\title{
Comments and Corrections
}

\section{Corrections on "Failure Transition Distance-Based Importance Sampling Schemes for the Simulation of Repairable Fault-Tolerant Computer Systems"}

Juan A. Carrasco
Index Terms-Fault-tolerant computer systems, importance sampling, Markov models, rare event simulation, steady-state availability, variance reduction.

The following corrections are to be made to [1]. The corrections do not propagate to any other part of the paper and do not affect the correctness of the experimental results reported in the paper.

- The definition of repair transition on page 208, right column should be:

repair transition a transition $(x, y)$ of $X(\Pi)$ is a repair transition if $F(y) \subset F(x)$

- The definition of $f_{x, y}, d_{x, y}$ in page 209 , left column should be:

$f_{x, y}, d_{x, y} \quad$ failure transitions $(x, y) \in T_{F}$ are modeled as $\lambda_{x, y}=r_{\min } f_{x, y} \varepsilon^{d_{x, y}}$, where $d_{x, y}$ is the minimum integer $\geq 1$ such that $f_{x, y} \gg \varepsilon$

- The definition of $o\left(\varepsilon^{d}\right)$ in page 209, right column should be:

$o\left(\varepsilon^{d}\right) \quad$ a function $f(\varepsilon), \varepsilon>0$ is said to be $o\left(\varepsilon^{d}\right)$ (written $\left.f(\varepsilon)=o\left(\varepsilon^{d}\right)\right)$, where $d$ is an integer $\geq 0$, if $\lim _{\varepsilon \rightarrow 0} f(\varepsilon) / \varepsilon^{d}=0$

- The definition of $\Theta\left(\varepsilon^{d}\right)$ in page 209 , right column should be:

$\Theta\left(\varepsilon^{d}\right)$ a function $f(\varepsilon), \varepsilon>0$ is said to be $\Theta\left(\varepsilon^{d}\right)$ (written $f(\varepsilon)=\Theta\left(\varepsilon^{d}\right)$ ), where $d$ is an integer $\geq 0$, if $f(\varepsilon)=c \varepsilon^{d}+o\left(\varepsilon^{d}\right)$ for some constant $c>0$

- In page 211 , right column, lines 27-28 $F(x)$ "

"\& repair transitions $(x, y)$, characterized by $F(y) \subseteq$ should be

" \& repair transitions $(x, y)$, characterized by $F(y) \subset$ $F(x)$ "

- In page 212 , left column, lines 22-24

"will model failure transition rates as $\lambda_{x, y} r_{\min } f_{x, y} \varepsilon^{d_{x, y}}$, $f_{x, y} \in(0,1], f_{x, y} \gg \varepsilon, d_{x, y} \geq 1 "$

should be

"will model failure transition rates as $\lambda_{x, y}=$ $r_{\min } f_{x, y} \varepsilon^{d_{x, y}}$, where $d_{x, y}$ is the minimum integer $\geq 1$ such that $f_{x, y} \gg \varepsilon "$

- In page 217 , left column, lines 13-15

“i.e. $\left\{(r, y) \in T_{F}(r), y \in D\right\} \neq \emptyset \& d_{r, y}=1$ for every $(r, y) \in T_{F}(r), y \in D^{\prime \prime}$

Manuscript received March 24, 2007. Associate Editor: J. Rupe.

The author is with the Departament d'Enginyeria Electrònica, Universitat Politècnica de Catalunya, Barcelona 08028, Spain (e-mail: carrasco@eel.upc. edu).

Digital Object Identifier 10.1109/TR.2007.896684 should be

"i.e. $\left\{(r, y) \in T_{F}(r), y \in D\right\} \neq \emptyset \& d_{r, y}=1$ for some

$(r, y) \in T_{F}(r), y \in D$ "

- The second paragraph in page 230 , left column should be

"Discarding minimal failure covers of cardinality $>M$ could allow us to deal with very large systems when $M$ is small. Thus, for instance, for $M=2$, the number of minimal failure covers of cardinality $\leq M$ of a system having $N C$ component classes with an instance of each class and with failure bags of cardinality 1 is roughly bounded from above by $N C^{2} / 2$, and a budget of 50,000 minimal failure covers would allow us to deal with systems with at least 316 components, and often many more. For $M=3$, the number of minimal failure covers with cardinality $\leq M$ is roughly bounded from above by $N C^{3} / 6$, and a budget of 50,000 minimal failure covers would allows us to deal with systems with at least 66 components, and often many more."

- In page 232, right column, line 5

"Proof of Theorem 6"

should be

"Proof of Theorem 1"

\section{REFERENCES}

[1] J.A. Carrasco, "Failure transition distance-based importance sampling schemes for the simulation of repairable fault-tolerant computer systems," IEEE Trans. Reliability, vol. 55, no. 2, pp. 207-236, June 2006.

\section{Erratum}

M. Kaminskiy, V. Krivtsov, and S. Nadarajah

In [1], $\Gamma\left(n_{0}\right)$ in the denominator of (6) should be replaced by $\Gamma\left(n_{0}-\right.$ $\left.x_{0}\right)$. Otherwise, this typo causes $\Gamma\left(n_{0}\right)$ to cancel in the numerator and the denominator of the mentioned equation. Thus, (6) in [1] should have appeared as

$$
f(\lambda)=t \frac{\Gamma\left(n_{0}\right)}{\Gamma\left(x_{0}\right) \Gamma\left(n_{0}-x_{0}\right)}\left[z^{n_{0}-x_{0}}(1-z)^{x_{0}-1}\right],
$$

where $z=e^{-t \lambda}$ and $\lambda>0$, and the respective expression for the expectation as

$E(\lambda)=t \frac{\Gamma\left(n_{0}\right)}{\Gamma\left(x_{0}\right) \Gamma\left(n_{0}-x_{0}\right)} \int_{0}^{\infty} \lambda\left[e^{-\left(n_{0}-x_{0}\right) \lambda t}\left(1-e^{-\lambda t}\right)^{x_{0}-1}\right] d \lambda$.

\section{REFERENCES}

[1] M. P. Kaminskiy and V. V. Krivtsov, "A simple procedure for Bayesian estimation of the Weibull distribution," IEEE Trans. Reliability, vol. 54, pp. 612-616, 2005.

Manuscript received March 24, 2007. Associate Editor: J. Rupe.

M. Kaminskiy is with the University of Maryland at College Park, USA.

V. Kristov is with the Ford Motor Company, Dearborn, USA.

Digital Object Identifier 10.1109/TR.2007.896683 